\title{
A Fast adaptive transmit power and bit allocation in OFDM system
}

\author{
Juan Wen ${ }^{1,}$, Qiming Tian ${ }^{2, b}$ \\ ${ }^{1,2}$ Dept. of Computer, Wenzhou Vocational \& Technical College, Wenzhou, China \\ aemail: wenjuan.726@163.com, bemail: tqm78@126.com
}

Keywords: OFDMA, bit allocation, BSL, optimal solution

\begin{abstract}
In this paper, we propose a fast and optimal adaptive transmit power and bit allocation algorithm, which is called Bisection Searching Level (BSL) for wireless OFDMA systems. The algorithm aims at maximization of data rate under the constraints of total transmit power and bit error rate (BER). BSL is based on water pouring approach, and adopt bisection algorithm to find the water pouring level under discrete bit restriction. Both theoretical analysis and simulation results demonstrate the optimality of our proposed algorithm.
\end{abstract}

\section{Introduction}

Recently, intense interests have been focused on the multicarrier modulation due to the demand for high-speed and reliable data transmission over multipath fading channels, which will introduce intersymbol interference (ISI) as a major problem. Orthogonal Frequency Division Multiplexing (OFDM) attracts much attention because of its high spectral efficiency and capability in combating frequency selective fading. In OFDMA systems, due to the different frequency selective fading experienced by each user, the channel gain varies from user to user at each subcarrier, and among all the subcarriers of a user [3]. Based on this characteristic, subcarriers allocation optimization can be made between users considering the subcarriers as a controllable resource.

The well-known water pouring method can be used distributing transmit power to the subcarriers together with the bit allocated, Unfortunately, considering the restriction of integer bit constellation in practice, there has been tremendous research on subcarrier and bit allocation in multiuser OFDM systems under the discrete bit restriction recently. In [1],Hughes-Hartogs developed a greedy algorithm achieving the optimal performance under the requirements of data rate and BER, however is computational costly. Jiho Jang in[2] proposed a simple and efficient but suboptimal loading algorithm to adaptively allocate bit and transmit power for each subcarrier.

In this paper, we propose a Bisection Searching Level (BSL) method aiming at maximization of data rate under the constraint of total transmit power and bit error rate (BER), which is drew inspiration from the Jiho Jang's. The BSL method has the same optimal performance as the greedy algorithm from an analytical view, while maintains the same computational complexity as the Jiho Jang's algorithm.

\section{Problem formulation}

Consider the OFDM-TDMA scheme, assume that the subcarriers are already adaptively allocated, then the user can use all subcarriers in the assigned time slot(s). Hence the resource allocation problem transforms to the distribution of the bit and transmit power, which can be categorized as two classes: Margin Adaptive(MA) optimization and Rate Adaptive(RA) optimization[4]. As they are counterparts, we just address the second one.

Assume that the total available bandwidth is divided into K subcarriers, and all the subcarriers will be allocated to a predetermined user at one slot and at the same time, all the channel gain $h_{k}$

$(\mathrm{k}=1,2, \ldots \ldots \mathrm{K})$ is also available in the transmitter. Here we consider the channel is quasi-static, that is, the channel remains unchanged during one transmission.

The number of loaded bits, $b_{k}$ for the kth subcarrier can be obtained by [5] 


$$
b_{k}=\log _{2}\left(1+\frac{h_{k} p_{k}}{\mathrm{~T} \sigma^{2}}\right)
$$

Accordingly, the transmit power allocated to the kth subcarrier $p_{k}$ is given by

where $\Gamma$ is the SNR gap [6].

$$
p_{k}=\frac{\Gamma \sigma^{2}}{h_{k}}\left(2^{b_{k}}-1\right)
$$

Consequently, the problem considered in this paper can be formulated as

$$
\begin{gathered}
\operatorname{Max} \sum_{k=1}^{K} b_{k} \\
\text { Subject to } \operatorname{BER} \leq \mathrm{BER}_{\text {target }} \text { and } \sum_{k=1}^{K} p_{k} \leq P_{T}
\end{gathered}
$$

where $P_{T}$ is the total available transmit power budget.

\section{Bisection Searching Level (BSL) method}

Bisection algorithm is a high efficient searching method, we can employ it with finding the water pouring level if the upper and lower bounds are known under the restriction of integer bit constellation in practice.

BSL method first adopt the water pouring algorithm to get the allocated bit and transmit power for each subcarrier, hence

$$
b_{k}=\left[\log _{2}\left(\lambda h_{k}\right)\right]^{+}, \quad x^{+}= \begin{cases}x & x>0 \\ 0 & x \leq 0\end{cases}
$$

Then we round off to make the $b_{k}$ be an integer number, consequently the total transmit power declined simultaneously. The water pouring level must be driven up so as to get the optimal solution. The lower bound can adopt the same as continues one [7],

$$
\lambda_{\min }^{d}=\frac{1}{K}\left(\frac{P_{T}}{\Gamma \sigma^{2}}+\frac{K}{h_{k^{*}}}\right), k^{*}=\arg \max _{k} h_{k} \text {. }
$$

Then, we round the $b_{k}$ to find the maximal increment to get the upper bound $\lambda_{\max }^{d}$, which is $\lambda_{\max }^{d}=\lambda_{\max }+\max _{k}\left\{\frac{1}{h_{k}}\left(2^{\left\lceil b_{k}\right\rceil}-2^{b_{k}}\right)\right\}$. It is easy to verify that the total transmit power is always larger than $P_{T}$ when using $\lambda_{\max }^{d}$ as the water pouring level.

The followings are the process of the proposed BSL algorithm.

(Step1) Set $m=\lambda_{\text {min }}^{d}, n=\lambda_{\text {max }}^{d}$. for $k=1,2, \cdots \cdots K$,calculate the loading bits

$b_{k}=\left\lfloor\log _{2}\left(\lambda_{\max }^{d} h_{k}\right)\right\rfloor^{+}$, then compute the total loading bits with the upper bound case $b_{u}=\sum_{k=1}^{K} b_{k}$.

(Step2): Set $l=\frac{m+n}{2}$, re-calculate $b_{k}=\left\lfloor\log _{2}\left(l h_{k}\right)\right\rfloor^{+}$, reckon the temporary total loading bits and the overall transmit power $b_{T}=\sum_{k=1}^{K} b_{k}, \quad P=\sum_{k=1}^{K} \frac{\Gamma \sigma^{2}}{h_{k}}\left(2^{b_{k}}-1\right)$ respectively.

(Step3): if $P>P_{T}$, namely, the water pouring level $l$ is too large for the power budget, we have to pull it down by updating $n=l, b^{u}=b_{T}$, GOTO step2; otherwise, GOTO step4.

(Step4): if $b^{u}-b_{T} \leq 1$, update $m=l$, GOTO step2; otherwise, mark the temporarily $l$ with $\lambda^{*}$ and jump out.

It is easy to prove that the BSL method satisfies two conditions given in [8] to be an optimal solution as the greedy algorithm. The necessary and sufficient conditions are presented as follows: 
C1. $\Delta p_{n}\left(b_{n}-1\right) \leq \Delta p_{m}\left(b_{m}\right), \forall n, m=1,2, \cdots, K$

C2. $0 \leq P_{T}-\sum_{k=1}^{K} p_{k}\left(b_{k}\right)<\min _{k} \Delta p_{k}\left(b_{k}\right)$ (aiming at the RA optimization), or $\sum_{k=1}^{K} b_{k}=B_{T}$ (aiming at the MA optimization )。

\section{Numeric Simulations}

In this section, we evaluate the performance of the BSL algorithm in terms of loading bits and required number of iterations by computer simulation. In simulation, the number of the subcarriers $\mathrm{K}$ is set to 256, and we assume that the OFDM symbols undergo Rayleigh fading. The average AWGN is $1 \mathrm{~mW}$ for each subcarrier. Assume that no channel coding scheme is used, and the target BER is $B E R_{\text {target }}=10^{-3}$, then the SNR gap $\Gamma$ is $5.48 \mathrm{~dB}$.

The performance measure of transmit power and bit allocation algorithms in OFDM systems is the required number of iterations and the average loading bits. Figure 1 shows the required iteration numbers of three bit loading algorithm, the greedy algorithm requires far more iterations than BSL and Jiho Jang's algorithm, and the gap gets higher when the SNR gets larger.

Observed from Fig2, the average loading bits are increasing according to the average SNR. The BSL reaches exactly the same average loading bits as the greedy algorithm, which is proved to be optimal solution, while the Jiho Jang's is the suboptimal.

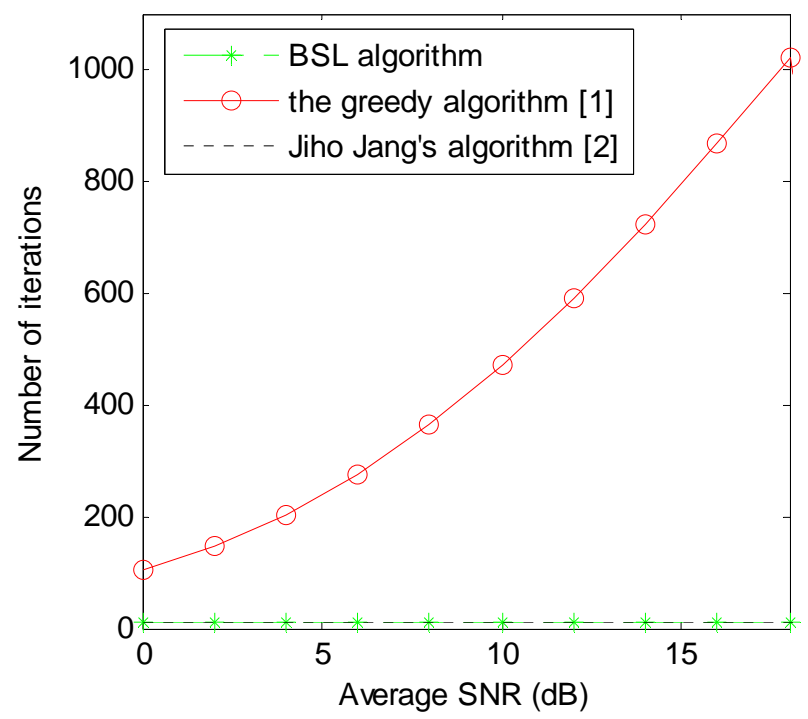

Fig.1.Required number of iterations for three algorithms 


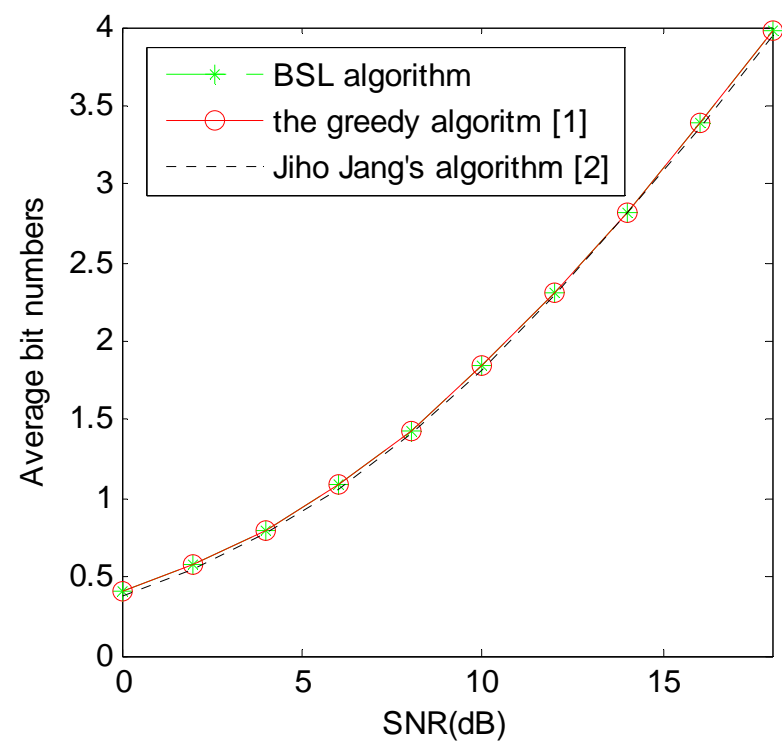

Fig.2.Average allocated bit numbers for three algorithms

\section{Conclusions}

In this paper, we propose a fast and optimal adaptive transmit power and bit allocation algorithm, which is based on the well-known water pouring approach, for the wireless OFDM systems. Owing to the efficient bisection algorithm, the proposed algorithm is computational efficient, and is demonstrated to be optimal solution in theory as well as in simulation.

\section{References}

[1] D. Hughes-Hartogs, Ensemble modem structure for imperfect transmission media, U.S. Patent 4,833,706. (1989)

[2] J. Jiho, L. K. Bok, Y. H. Lee, Transmit power and bit allocations for OFDM systems in a fading channel, GLOBECOM '03. San Francisco, USA, 2003, 2: 858 - 862.

[3] Y. W. Cheong, R. S. Cheng, K. B. Letaief, and R. D. Murch, Multiuser OFDM with adaptive subcarrier, bit, and power allocation, IEEE J. Select. Areas Commun., 17(1999)1747-1758.

[4] I. Kim, H. L. Lee, B. Kim, et al, On the use of linear programming for dynamic subchannel and bit allocation in multiuser OFDM, in Proc. IEEE GLOBECOM’01, San Antonio, Texas, 2001, 1: 3648-3652.

[5] P. S. Chow, Bandwidth optimized digital transmission techniques for spectrally shaped channels with impulse noise, Ph.D.Thesis, Stanford University, 1993.

[6] G. D. Forney Jr. and M.V. Eyuboglu, Combined Equalization and Coding Using Precoding. IEEE Communications Magazine, 29(1991)25-34.

[7] G. D. Yu, Z. Y. Zhang and P. L. Qiu, Bit and Power Allocation Algorithm for OFDM System, Journal of Electronics and Information Technology, 27(2005)1479-1482. 